\title{
Essential Fatty Acids for Childhood Mental Health Disorders
}

Barbara L. Gracious, M.D. ${ }^{1, *}$ Lipi Gupta, B.A. ${ }^{2,4}$

L. Eugene Arnold, M.D., M.Ed. ${ }^{3}$

\author{
Address \\ ${ }^{*}, 1$ Center for Innovation in Pediatric Practice, The Research Institute at Nation- \\ wide Children's Hospital and The Ohio State University, 700 Children's Dr., JWest \\ 4923, Columbus, OH 43205, USA \\ Email: Barbara.gracious@nationwidechildrens.org \\ ${ }^{2}$ The Ohio State University Medical Center, 1670 Upham Dr., Columbus, OH 43210, \\ USA \\ ${ }^{3}$ The Ohio State University Medical Center, Nisonger Center, The Ohio State \\ University, 1581 Dodd Dr., Columbus, OH 43210, USA \\ ${ }^{4} 7101$ Churchill Rd, McLean, VA 22101, USA
}

Published online: 10 February 2015

(C) Springer International Publishing AG 2015

This article is part of the Topical Collection on Child and Adolescent Psychiatry

Keywords Complementary and alternative medicine - 0mega-3 supplementation - Children - Adolescents · Recommendation - Hyperactivity - Aggression - Dyslexia - Dyspraxia - Tourette's Disorder - Autism - Psychosis . Behavior

\section{Opinion statement}

No child psychiatric disorder has a single treatment that is completely satisfactory. Combinations are sometimes more beneficial than single treatments. This is well established for medication+behavioral treatment for depression and ADHD. There is wide variability in the evidence base for various treatments, from FDA-approved RCTs to open pilots. In the search for additional or alternative treatments, essential fatty acids seem especially to pass the SECS criterion: a treatment that is safe, easy, cheap, and sensible does not need as much evidence to justify patient trials as one that is risky, unrealistic, difficult, or expensive (RUDE). Not only do omega-3 fatty acids have some RCT evidence for a small effect in several psychiatric disorders, but they also are believed useful in preventing cardiac morbidity and excessive inflammation.

Therefore, for $\mathrm{ADHD}$, we recommend a combination of behavioral treatment (e.g., parent training, cognitive-behavioral therapy (CBT) for older patients), FDA-approved medication, (primarily stimulants), an RDI/RDA multivitamin/mineral to compensate for stimulant appetite suppression, and about a gram a day of EPA/DHA, possibly supplemented with 50-100 mg GLA.

For mood disorders, we recommend a combination of mood stabilizer or antidepressant 
(depending on which mood disorder), CBT, RDA/RDI multivitamin/mineral (in view of reported vitamin $D$ deficiencies and vitamin wasting from some anticonvulsant mood stabilizers), and a gram per day of EPA/DHA.

For autism, we recommend applied behavior analysis, RDA/RDI multivitamin/mineral in view of the often idiosyncratic unbalanced diet, ADHD medication if a problem with hyperactivity, antipsychotic if a problem with irritability/aggression, and omega-3 for anti-inflammatory effect.

For learning disorder and developmental coordination disorder, we recommend remedial tutoring or occupational therapy and EPA/DHA.

For children at high risk for psychosis or mood disorder, we recommend counseling, monitoring for early indication of low-dose antipsychotic, and general nutritional support, including EPA/DHA.

For Tourette's and aggression, EPA/DHA and other nutritional support can be added to standard treatment.

When using fish oil, it is important to make sure it was refined to eliminate contaminants such as mercury. The effect accumulates gradually over months. There is no convincing evidence of greater benefit but some risk from amounts over a gram or two per day.

\section{Introduction}

\section{Brief history of childhood psychiatric illnesses}

As many as $20 \%$ of the world's children may be affected by a psychiatric or neurodevelopmental disorder [1]. Their families may seek complementary and alternative (CAM) treatments as psychotropic providers and treatments may not be available; financial resources, cultural acceptance, or concern about potential adverse side effects or developmental consequences of conventional established treatment also influence desire to use a CAM modality.

\section{Overview of current treatment options}

For the disorders reviewed here, the conventional psychopharmacologic treatments (psychostimulants, antidepressants, antipsychotics, and classic mood stabilizers) are limited by response rates and tolerability, which are both unpredictable. Tolerability concerns include the following: appetite and weight changes; sedation and dizziness; bleeding, blood pressure, and heart rate changes [2]; alterations in sleep; and undesired changes in mood, or worsening of or emergence of suicidal ideation. Adverse effects can be subclinical when viewed at individual visits but over time may build to serious clinical sequelae such as type 2 diabetes, cardiovascular disease, or neurological impairment. Thus, effective and better tolerated options are critical to improving outcomes of childhood psychiatric and neurodevelopmental disorders. CAM may also offer population health strategies for preventing these disorders.

Of the many potential CAM treatments being examined for prevention and intervention in child and adolescent disorders, omega-3 polyunsaturated fatty acid (PUFA) supplementation is one of the most promising, with supportive data for basic and clinical mechanism of action and epidemiologic studies. Randomized controlled trials (RCTs) are building evidence of benefit for several disorders, as well as for maximizing developmental potential and preventing brain-based disorders as well as associated metabolic health sequelae $[3,4$, $5 \bullet]$.

\section{Roles and possible mechanisms of omega-3 PUFAs}

Essential fatty acids (omega-3 and omega-6 PUFAs) are obtained only through diet. Omega-3 PUFAs, chiefly docosahexaenoic acid (DHA), are necessary during pregnancy, infancy, and early childhood for appropriate development and function of the central nervous system. With adequate dietary intake, over $20 \%$ of brain lipid is DHA, and combined with another fraction as eicosapentaenoic acid (EPA), both contribute to neuronal cell membrane structure, fluidity, and receptor function. The decline of dietary ratios of omega-3:omega-6 PUFAs across the last 
century have very possibly contributed to greater rates

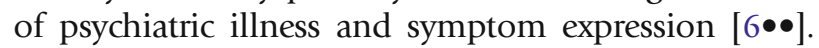

Omega-3 PUFAs are linked to dopaminergic function in adults with major depressive disorder [7]. They also help regulate inflammation. Eicosanoids, signaling molecules derived from omega-3 and omega-6 PUFAs, include prostaglandins and leukotrienes. Series 2 eicosanoids, from omega-6 (arachidonic acid, AA), are proinflammatory, and series 1 and 3 (from omega-6 (dihomogammalineolenic acid, GLA) and from omega-3 (eicosapentaenoic acid, EPA) are mainly anti-inflammatory. Inflammation plays a role in many pathophysiological conditions [8] including the psychiatric and neurodevelopmental disorders listed below, providing potential mechanisms for study. The studies listed below include primarily omega-3 FA RCTs.

\section{Summary of cost-effectiveness}

There are not yet sufficient reports to determine costeffectiveness of treatment or prevention effects to make a definitive statement for childhood mental disorders, although the role of fish oils in population health (such as improved IQ) may be compelling for promotion of brain development during pregnancy [9•]. N-3 FA supplementation was safe and welltolerated in the trials reported below, without association to any serious adverse events to date or significant difference in tolerability from placebo, outside of the fish oil taste and odor when not masked. The most frequently reported adverse events have included difficulty swallowing large capsules, nausea, dyspepsia, vomiting, diarrhea, abdominal pain, and headaches, which were transient, self-limited, and equally reported in the groups taking placebo. Though no clinical trial has provided evidence for safety issues, there is a risk of contamination (mercury, other chemicals) in fish oil that is not purified.

Baseline and post-treatment serum markers from some omega-3 FA studies included plasma or RBC fatty acid concentrations, which can determine the extent to which subjects absorbed the oil (a potential compliance marker), or evidence of whether subjects converted alpha-linoleic acid (ALA) to bioavailable EPA/DHA.

The evidence justifying use is overall not conclusive for all children. A recurring theme through findings from treating children with omega-3 FAs is that certain children, depending on their n-3 FA dietary intake and other factors, such as genetic ability to convert DHA to EPA, may benefit more than others. In part related to limited efficacy of current pharmacologic treatments, their possibility of serious side effects, and the cost of adjunct omega-3 FA therapy as generally similar to or less than a monthly copay for conventional psychopharmacology, the American Psychiatric Association Task Force on Complementary and Alternative Medicine has recommended that for patients with poor impulse control, mood disorders, or psychotic disorders, the dietary intake of EPA+DHA should approximate 1 gram per day.

\section{0mega-3 fatty acid treatments in children}

\section{ADD/ADHD}

- $\quad \mathrm{N}-3$ supplementation to treat hyperactivity and inattention symptoms has a Grade $\mathrm{C}$ evidence base, according to standards established by the U.S. Preventive Services Task Force (USPSTF). There is moderate certainty of small benefit, based on at least 19 RCTs, one other trial, and four meta-analyses in a total of 1884 children.

- Of the 14 studies that measured biomarkers of n-3 FA composition, most found elevated cheek cell, serum, or RBC n-3 FA levels after treatment, at least marginally over control groups, which correlated with clinical improvement $[12-19,29-31,34]$. With the exception of one study [24], children with lower n-3/n-6 ratios at baseline exhibited better clinical response than the overall treated sample [13, 14, 19]. 
- Thirteen studies (using 11 datasets) reported significant improvement in inattention, cognition, or hyperactivity via a primary $[10-12,15,16$, $19,25-28,30,33$ ] or secondary measure [20]. However, evidence was limited by confounded and inconsistent treatment formulations that may have included EPA, DHA, LA, ALA, GLA, methylphenidate, phosphatidylserine, and micronutrients. The more responsive children included those with the following: comorbid learning difficulties [13, 14], comorbid neurodevelopmental disorders (motor control and perception) $[17,18]$, inattentive type ADHD [17, 18], oppositional symptoms [19], and more pronounced hyperactivity and impulsivity symptoms [20].

- N-3 FAs were better tolerated and only slightly less effective than methylphenidate $[21,25,26]$. Though the combination of methylphenidate and n-3 FAs offered no additional benefit over methylphenidate monotherapy, the combination allowed for slightly lower methylphenidate doses [25].

- Findings do not support the use of ALA or LA supplementation alone [22-24] while studies using a combination of EPA and GLA yielded the greatest treatment effects $[61 \bullet]$.

\section{Clinical evidence}

\begin{tabular}{|c|c|c|c|c|c|c|}
\hline Study & Type & $N$ & $\begin{array}{l}\text { Daily dose } \\
\text { /length }\end{array}$ & Outcome $^{a}$ & Statistic $^{a}$ & $\begin{array}{r}\text { Serum } \\
\text { data }\end{array}$ \\
\hline $\begin{array}{l}\text { [12] Widenhorn- } \\
\text { Muller et al., } \\
2014\end{array}$ & RCT & 95 & $\begin{array}{l}4 \mathrm{mo} .: 600 \mathrm{mg} \\
\text { EPA, } 120 \mathrm{mg} \\
\text { DHA, } 15 \mathrm{mg} \text { Vit E }\end{array}$ & $\begin{array}{l}\text { Working } \\
\text { memory }\end{array}$ & $\begin{array}{l}F=5.54 \\
\quad p=0.019^{h}\end{array}$ & $Y$ \\
\hline $\begin{array}{l}\text { [25] Barragán } \\
\text { et al., 2014/ } \\
\text { [26] Millichap, } \\
2014\end{array}$ & $\mathrm{RCT}^{\mathrm{f}}$ & 90 & $\begin{array}{l}12 \mathrm{mo.}: 558 \mathrm{mg} \\
\text { EPA, } 174 \mathrm{mg} \mathrm{DHA} \text {, } \\
60 \mathrm{mg} \mathrm{GLA}\end{array}$ & $\begin{array}{l}\text { Hyperactivity/ } \\
\text { Inattention }\end{array}$ & $\begin{array}{l}\text { Cohen's effect } \\
\text { size }=-2.68 \\
p<0.05^{\mathrm{h}}\end{array}$ & $N$ \\
\hline $\begin{array}{l}\text { [13] Milte } \\
\text { et al., 2013/ }\end{array}$ & $\mathrm{RCT}^{\mathrm{g}}$ & 90 & $\begin{array}{l}12 \mathrm{mo} .: 1109 \mathrm{mg} \text { EPA, } \\
108 \mathrm{mg} \text { DHA or } 264 \\
\mathrm{mg} \text { EPA } 1032 \mathrm{mq} \mathrm{DHA}\end{array}$ & Literacy & $p>0.05^{b}$ & Y \\
\hline $\begin{array}{l}\text { [14] Milte et al., } \\
2012\end{array}$ & $\mathrm{RCT}^{\mathrm{g}}$ & 90 & $\begin{array}{l}4 \text { mo.: } 1109 \mathrm{mg} \mathrm{EPA}, 108 \\
\text { mg DHA or } 264 \mathrm{mg} \text { EPA, } \\
1032 \mathrm{mg} \text { DHA }\end{array}$ & Literacy & $\begin{aligned} t=0.73 \\
p=0.47\end{aligned}$ & $Y$ \\
\hline $\begin{array}{l}\text { [27] Hariri et al., } \\
2012\end{array}$ & RCT & 103 & $\begin{array}{l}2 \mathrm{mo} .: 635 \mathrm{mg} \text { EPA, } 195 \\
\mathrm{mg} \mathrm{DHA}\end{array}$ & Hyperactivity & $p<0.05^{b, h}$ & Y \\
\hline [28] Perera et al., 2012 & RCT & 94 & $\begin{array}{l}6 \mathrm{mo} .: 1186 \mathrm{mg} \text { EPA, } 723 \\
\mathrm{mg} \text { DHA, methylphenidate } \\
0.7-1 \mathrm{mg} / \mathrm{kg}\end{array}$ & Inattention & $\begin{array}{l}\text { Effect size } \\
\quad=0.5877 \\
p<0.05^{\mathrm{h}}\end{array}$ & Y \\
\hline $\begin{array}{l}\text { [20] Manor et al., } \\
2012\end{array}$ & RCT & 200 & $\begin{array}{l}30 \text { wk: } 40-80 \mathrm{mgEPA}, \\
20-40 \mathrm{mg} \text { DHA, } 150- \\
300 \mathrm{mg} \text { phosphatidylserine }\end{array}$ & $\begin{array}{l}\text { Hyperactivity/ } \\
\text { Inattention }\end{array}$ & $p>0.05^{b}$ & $N$ \\
\hline $\begin{array}{l}\text { [19] Gustafsson } \\
\text { et al., } 2010\end{array}$ & RCT & 92 & 15 wk.: $500 \mathrm{mg}$ EPA & Inattention & $\begin{array}{l}\text { Effect size }=0.28, p= \\
0.04^{\mathrm{h}}\end{array}$ & $Y$ \\
\hline $\begin{array}{l}\text { [29] Kirby et al., } \\
2010\end{array}$ & RCT & 450 & $\begin{array}{l}4 \text { mo.: } 56 \mathrm{mg} \mathrm{EPA}, 400 \mathrm{mg} \text { DHA, } \\
\text { multivitamin }\end{array}$ & Inattention & $\begin{array}{l}F(1,245)=0.309 \\
p>0.05\end{array}$ & $N$ \\
\hline $\begin{array}{l}\text { [17] Johnson et al., } \\
\text { 2012/ } \\
\text { [18] Johnson } \\
\text { et al., } 2009\end{array}$ & RCT & 75 & $\begin{array}{l}6 \text { mo.: } 558 \mathrm{mg} \text { EPA, } 174 \mathrm{mg} \\
\text { DHA, } 60 \mathrm{mg} \text { n-6 linoleic acid, } \\
10.8 \mathrm{mg} \text { Vit E }\end{array}$ & Inattention & $p=0.14^{b}$ & $Y$ \\
\hline $\begin{array}{l}\text { [16] Belanger } \\
\text { et al., } 2009\end{array}$ & RCT & 37 & $\begin{array}{l}4 \text { mo.: } 500-1000 \mathrm{mg} \text { EPA } \\
(20-25 \mathrm{mg} / \mathrm{kg}), 200-400 \mathrm{mg} \\
\text { DHA }(8.5-10.5 \mathrm{mg} / \mathrm{kg}), \\
\text { 50-100 mg phospholipids, }\end{array}$ & $\begin{array}{l}\text { Hyperactivity/ } \\
\text { Inattention }\end{array}$ & $p<0.05^{b, h}$ & $Y$ \\
\hline
\end{tabular}




\begin{tabular}{|c|c|c|c|c|c|c|}
\hline & & & $\begin{array}{l}7.5-11.25 \\
\mathrm{mg} \text { Vit E }\end{array}$ & & & \\
\hline [22] Raz 2009 & RCT & 73 & 7 wk.: $120 \mathrm{mg}$ ALA, & Hyperactivity/ & $\begin{array}{c}F(1,61)=0.68 \\
D=0.413\end{array}$ & N \\
\hline $\begin{array}{l}\text { [30] Vaisman } \\
\text { et al., } 2008\end{array}$ & RCT & 83 & $\begin{array}{l}3 \text { mo.: } 156 \mathrm{mg} \text { EPA, } 95 \mathrm{mg} \text { DHA, } \\
300 \mathrm{mg} \text { phosphatidylserine or } \\
156 \mathrm{mg} \text { EPA, } 95 \mathrm{mg} \text { DHA, } 550 \\
\text { mg other FAs }\end{array}$ & Inattention & $\begin{aligned} p=0.413 \\
p<0.001^{b, h}\end{aligned}$ & Y \\
\hline $\begin{array}{l}\text { [10] Sinn et al., } 2008 \\
\text { /[11] Sinn } \\
\text { \& Bryan, } \\
2007\end{array}$ & RCT & 129 & $\begin{array}{l}30 \text { wk.: } 558 \text { mg EPA, } 174 \mathrm{mg} \mathrm{DHA} \text {, } \\
60 \mathrm{GLA} \text {, multivitamin }\end{array}$ & Inattention & $\begin{array}{c}F(1,126)=11.73 \\
\text { Cohen's Effect } \\
\text { Size }=0.43 \\
p=0.002^{\mathrm{h}}\end{array}$ & $N$ \\
\hline $\begin{array}{l}\text { [15] Joshi } \\
\text { et al. } 2006\end{array}$ & Trial $^{c, d, e}$ & 30 & $3 \mathrm{mo} .: 400 \mathrm{mg}$ ALA, $50 \mathrm{mg} \operatorname{Vit} \mathrm{C}$ & Hyperactivity & $t=4.6, p<0.001^{\mathrm{h}}$ & $Y$ \\
\hline $\begin{array}{l}\text { [32] Hiriyama } \\
\text { et al., } 2004\end{array}$ & RCT & 40 & 2 mo.: 514 mg DHA & Inattention & $p>0.05^{b}$ & $N$ \\
\hline $\begin{array}{l}\text { [34] Stevens } \\
\text { et al., } 2003\end{array}$ & RCT & 50 & $\begin{array}{l}4 \mathrm{mo} .: 80 \mathrm{mg} \text { EPA, } 480 \mathrm{mg} \text { DHA, } \\
96 \mathrm{mg} \\
\text { GLA, } 40 \mathrm{mg} \mathrm{AA}, 24 \mathrm{mg} \text { Vit } \mathrm{E}\end{array}$ & Disruptive behavior & $H=0.29, p=0.19$ & $Y$ \\
\hline $\begin{array}{l}\text { [33] Richardson } \\
\text { \& Puri } \\
\text { et al., } 2002\end{array}$ & RCT & 41 & $\begin{array}{l}3 \text { mo.: } 186 \text { mg EPA, } 480 \text { mg DHA, } \\
96 \text { mg } \\
\text { GLA, } 60 \text { IU Vit E, } 864 \text { mg cis- } \\
\text { linoleic } \\
\text { acid, } 42 \mathrm{mg} \mathrm{AA}\end{array}$ & $\begin{array}{l}\text { Hyperactivity/ } \\
\text { Inattention }\end{array}$ & $Z=2.21, p<0.03^{\mathrm{h}}$ & N \\
\hline [31] Voigt et al., 2001 & RCT & 63 & 4 mo.: $345 \mathrm{mg}$ DHA & Inattention & $p>0.05^{b}$ & $Y$ \\
\hline $\begin{array}{l}\text { [23] Arnold } \\
\text { et al. } 1989\end{array}$ & RCT & 18 & $\begin{array}{l}1 \text { mo.: } 2800 \text { mg LA, } 320 \text { GLA, } \\
104 \text { IU Vit E }\end{array}$ & $\begin{array}{l}\text { Hyperactivity/ } \\
\text { Inattention }\end{array}$ & $p>0.05^{b}$ & N \\
\hline $\begin{array}{l}\text { [24] Aman } \\
\text { et al.1987 }\end{array}$ & RCT & 31 & 8 wk.: $2160 \mathrm{mg}$ LA, $270 \mathrm{GLA}$ & Impulsivity & $\begin{aligned} F= & 0.001-0.372 \\
p & >0.05\end{aligned}$ & $Y$ \\
\hline $\begin{array}{l}\text { GLA gamma-linoleic a } \\
{ }^{a} \text { Primary clinical } \\
{ }^{\mathrm{b}} \text { No test statistic prov } \\
{ }^{\mathrm{c}} \text { Non-randomized } \\
{ }^{\mathrm{d}} \text { Non-placebo controll } \\
{ }^{\mathrm{e}} \text { Nonblinded } \\
{ }^{\mathrm{f}} \text { Non-placebo controll } \\
{ }^{\mathrm{g}} \text { Non-placebo controll } \\
{ }^{\mathrm{h}} \text { Statistically significa }\end{array}$ & $\begin{array}{l}\text { ALA alph } \\
\text { controlle } \\
\text { controlle } \\
\text { controlle }\end{array}$ & $\begin{array}{l}\text { th } 30 \\
\text { th } \mathrm{me} \\
\text { th } L A\end{array}$ & $\begin{array}{l}\text { e/gender-matched healthy control } \\
\text { lphenidate pharmacotherapy } \\
\text { noleic acid) } 1467 \mathrm{mg}\end{array}$ & jects & & \\
\hline
\end{tabular}

\section{Tourette's disorder}

- There is insufficient evidence to determine efficacy of n-3 FAs for treating tics for children with Tourette's Disorder. In the single pertinent published RCT to date, n-3 FA supplementation did not reduce tic severity (number, intensity, frequency, complexity, and interference); however, it did reduce overall tic-related impairment on self-esteem, school, family, and social functioning [35].

\section{Clinical evidence}

\begin{tabular}{|c|c|c|c|c|c|c|}
\hline Study & Type & $N$ & Daily dose/length & Outcome $^{a}$ & Statistic $^{a}$ & Serum \\
\hline $\begin{array}{l}\text { [35] Gabbay } \\
\text { et al., } 2012\end{array}$ & $\mathrm{RCT}$ & 33 & $\begin{array}{l}20 \text { wk.: } 334-4000 \mathrm{mg} \\
\text { EPA (titrated), 167- } \\
2000 \mathrm{mg} \mathrm{DHA} \text { (titrated) }\end{array}$ & $\begin{array}{l}\text { Global Tic Severity S } \\
\text { cale-impairment }\end{array}$ & $t=0.69, p>0.1^{a}$ & \\
\hline
\end{tabular}




\section{Dyspraxia/developmental coordination disorder}

- There is insufficient evidence to determine efficacy of n-3 FAs for treating motor impairment that is not due to a generalized medical condition (e.g. cerebral palsy). To date, one small trial reports improved motor function after DHA and AA supplementation, a positive finding that awaits confirmation. The single published RCT of children with developmental coordination disorder (DCD) reported a treatment effect, not on motor impairment, but on reading, spelling, and ADHD-related behavioral symptoms [36], consistent with other studies done with samples with dyslexia [38] and ADHD [18].

\section{Clinical evidence}

\begin{tabular}{|c|c|c|c|c|c|c|}
\hline Study & Type & N & Daily dose/length & Outcome $^{a}$ & Statistic ${ }^{a}$ & Serum \\
\hline $\begin{array}{l}\text { [36] Richardson, } \\
2005\end{array}$ & RCT & 117 & $\begin{array}{c}3 \text { mo.: } 558 \mathrm{mg} \text { EPA, } \\
174 \mathrm{mg} \text { DHA, } 60 \mathrm{mg} \\
\mathrm{GLA}, 9.6 \mathrm{mg} \text { Vit } \mathrm{F}\end{array}$ & Motor impairment & $Z=0.42, p=0.7$ & N \\
\hline $\begin{array}{l}\text { [37] Stordy, } \\
2000\end{array}$ & Trial $^{\mathrm{d}}$ & 17 & $\begin{array}{l}4 \mathrm{mo.:} 480 \mathrm{mg} \text { DHA, } \\
35 \mathrm{mg} \mathrm{AA}, 96 \mathrm{mg} \mathrm{GLA} \text {, } \\
80 \mathrm{mg} \text { Vit E }\end{array}$ & Motor impairment & $p<0.001^{\mathrm{b}, \mathrm{e}}$ & N \\
\hline \multicolumn{7}{|c|}{$\begin{array}{l}\text { GLA gamma-linoleic acid, } A A \text { arachidonic acid } \\
\text { a Primary clinical } \\
{ }^{\mathrm{b}} \text { No test statistic provided } \\
{ }^{c} \text { Non-randomized } \\
{ }^{\mathrm{d}} \text { Non-controlled } \\
{ }^{\text {e Statistically significant }}\end{array}$} \\
\hline
\end{tabular}

\section{Dyslexia and cognitive functioning}

- There is insufficient evidence to determine treatment efficacy of n-3 FAs for treating dyslexia, reading problems, and other learning disabilities. The RCT focused on children with dyslexia provides no evidence of reading improvement [40]. Two of the three RCTs evaluating healthy children $[38,39,41]$ found mild benefit in reading fluency [38] and listening comprehension [39].

\section{Clinical evidence}

\begin{tabular}{|c|c|c|c|c|c|c|}
\hline Study & Type & $N$ & Daily dose/length & Outcome $^{a}$ & Statistic $^{a}$ & Serum \\
\hline $\begin{array}{l}\text { [40] Kairaluoma } \\
\text { et al. } 2009\end{array}$ & RCT & 61 & $\begin{array}{l}3 \text { mo.: } 500 \text { mg EPA, } \\
400 \mathrm{mq} \text { carnosine }\end{array}$ & Reading & $\begin{array}{c}F(1,58)=0.045, \\
n=0.833\end{array}$ & $y$ \\
\hline $\begin{array}{l}\text { [38] Richardson } \\
\text { et al., } 2012\end{array}$ & RCT & 362 & 4 mo.: $600 \mathrm{mg} \mathrm{DHA}$ & $\begin{array}{l}\text { Reading } \\
\text { fluency }\end{array}$ & $p>0.05^{\mathrm{b}}$ & $N$ \\
\hline \multirow[t]{2}{*}{$\begin{array}{l}\text { [41] Ryan \& } \\
\text { Nelson, } 2008\end{array}$} & RCT & 175 & 4 mo.: 400 mg DHA & Attention & $p>0.05^{b}$ & Y \\
\hline & RCT & 780 & & & & $\gamma$ \\
\hline
\end{tabular}




\begin{tabular}{|c|c|c|c|}
\hline $\begin{array}{l}\text { [39] Osendarp } \\
\text { et al., } 2007\end{array}$ & $\begin{array}{l}12 \text { mo.: } 88 \mathrm{mg} \text { DHA, } \\
\text { multivitamin/ } 88 \mathrm{mg} \\
\text { DHA, } 22 \mathrm{mp} \text { EPA, multivitamin/ } \\
22 \mathrm{mg} \text { EPA, multivitamin }\end{array}$ & $\begin{array}{l}\text { Auditory verbal } \\
\text { learning }\end{array}$ & $\begin{array}{c}\text { Cohen's effect size }=-0.04 \\
-0.11, p=0.66,0.63\end{array}$ \\
\hline
\end{tabular}

- There is insufficient evidence to determine efficacy of n-3 FAs on autism. To date, results of five RCTs have been published with children with autism and provide no evidence of reduction in behavioral and developmental outcomes. However, most of the studies were underpowered.

\section{Clinical evidence}

\begin{tabular}{|c|c|c|c|c|c|c|}
\hline Study & Type & $N$ & Daily dose/length & Outcome $^{a}$ & Statistic ${ }^{a}$ & Serum \\
\hline [42] Voigt et al., 2014 & RCT & 48 & 3 mo.: $200 \mathrm{mg}$ DHA & $\begin{array}{l}\text { Global } \\
\text { improvement }\end{array}$ & $p=0.04^{b}$ & $y^{\text {uata }}$ \\
\hline [43] Bent et al., 2014 & RCT & 57 & $\begin{array}{c}6 \text { wk.: } 700 \mathrm{mg} \mathrm{EPA} \text {, } \\
460 \mathrm{mg} \text { DHA }\end{array}$ & Hyperactivity & $\begin{array}{l}\text { Effect size }=0.26 \\
\quad p=0.38\end{array}$ & N \\
\hline [44] Bent et al., 2011 & RCT & 27 & $\begin{array}{c}3 \text { mo.: } 700 \mathrm{mg} \mathrm{EPA} \text {, } \\
460 \mathrm{mg} \text { DHA }\end{array}$ & Hyperactivity & $\begin{array}{l}\text { Effect size }=0.38 \\
\quad p=0.40\end{array}$ & N \\
\hline $\begin{array}{l}\text { [45] Johnson et al., } \\
2010\end{array}$ & $\mathrm{RCT}^{\mathrm{e}}$ & 23 & 3 mo.: 400 mg DHA & $\begin{array}{l}\text { Total behavior } \\
\text { rating }\end{array}$ & $\begin{aligned} F=0.441 \\
p=0.514\end{aligned}$ & N \\
\hline & $\mathrm{CT}^{\mathrm{C}}$ & 30 & $\begin{array}{c}3 \text { mo.: } 240 \mathrm{mg} \text { EPA, } 52 \mathrm{mg} \mathrm{DHA} \text {, } \\
\text { unspecified amount of Vit } \mathrm{E}\end{array}$ & $\begin{array}{l}\text { Total behavior } \\
\text { rating }\end{array}$ & $p<0.0001^{b, f}$ & $Y$ \\
\hline $\begin{array}{l}\text { [47] Amminger et al., } \\
2007\end{array}$ & & 13 & $\begin{array}{l}6 \text { wk.: } 840 \mathrm{mg} \mathrm{EPA}, 700 \mathrm{mg} \text { DHA, } \\
7 \mathrm{mg} \text { Vit E }\end{array}$ & Hyperactivity & $\begin{array}{l}\text { Glass' effect size }=0.71, \\
\quad p>0.05\end{array}$ & N \\
\hline [48] Meiri et al., 2009 & Trial ${ }^{\mathrm{c}, \mathrm{d}, \mathrm{e}}$ & 10 & 3 mo.: $380 \mathrm{mg} \mathrm{EPA}, 180 \mathrm{mg}$ DHA & $\begin{array}{l}\text { Total behavior } \\
\text { rating }\end{array}$ & $p>0.05^{b}$ & N \\
\hline \multicolumn{7}{|c|}{$\begin{array}{l}{ }^{a} \text { Primary clinical } \\
{ }^{b} \text { No test statistic provided } \\
\text { cNon-randomized } \\
{ }^{d} \text { Non-controlled } \\
\text { eNonblinded } \\
\text { 'N Statistically significant }\end{array}$} \\
\hline
\end{tabular}

\section{Psychosis and personality disorders}

- There is insufficient evidence to determine efficacy of n-3 FAs on

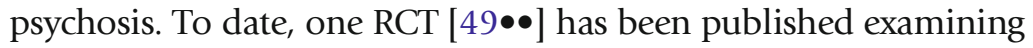
clinical symptoms of adolescents and young adults with subthreshold psychosis and borderline personality disorders. It, along with a posthoc analysis [50] and enzymatic study [51], provides suggestive but insufficient evidence. Large, well-designed randomized controlled trials are required to determine the clinical efficacy of n-3 FA supplementation for youth at risk for developing their first psychotic episode. 


\section{Clinical evidence}

\begin{tabular}{|c|c|c|c|c|c|c|}
\hline 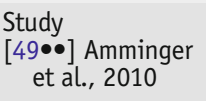 & $\begin{array}{l}\text { Type } \\
\text { RCT }\end{array}$ & $\begin{array}{l}N \\
81\end{array}$ & $\begin{array}{l}\text { Daily dose/length } \\
4 \mathrm{mo} .: 700 \mathrm{EPA}, 480 \mathrm{DHA} \text {, } \\
\quad 7.6 \mathrm{mg} \text { Vit E, } 220 \mathrm{mg} \text { other } \mathrm{n}-3 \mathrm{FAs}\end{array}$ & $\begin{array}{l}\text { Outcome }^{\mathrm{a}} \\
\text { Time to transition } \\
\text { to psychotic disorder }\end{array}$ & $\begin{array}{l}\text { Statistic } \\
x^{2}=7.32 \\
p=0.007^{b}\end{array}$ & $\begin{array}{l}\text { Serum data } \\
Y\end{array}$ \\
\hline
\end{tabular}

\section{Aggression}

- To date, two published RCTs have assessed efficacy in reducing aggression among both healthy schoolchildren [52] and those and with disruptive behavior disorders [53]. These provide suggestive but insufficient evidence. Additional large, well-designed randomized controlled trials are required.

\section{Clinical evidence}

\begin{tabular}{|c|c|c|c|c|c|c|}
\hline $\begin{array}{l}\text { Study } \\
\text { [52] Dean et al., } 2014 \\
\text { [53] Itomura et al., } 2005\end{array}$ & $\begin{array}{l}\text { Type } \\
\text { RCT } \\
\text { RCT }\end{array}$ & $\begin{array}{l}N \\
21 \\
166\end{array}$ & $\begin{array}{l}\text { Daily dose/length } \\
4 \mathrm{mo} .: 400 \mathrm{mg} \mathrm{EPA}, 2000 \mathrm{mg} \text { DHA } \\
3 \mathrm{mo} .: 840 \mathrm{mg} \text { EPA, } 3600 \mathrm{mg} \text { DHA }\end{array}$ & $\begin{array}{l}\text { Outcome }^{a} \\
\text { Aggression } \\
\text { Aggression }\end{array}$ & $\begin{array}{l}\text { Statistic }{ }^{\mathrm{a}} \\
F(1,19)=0.05, p=0.82 \\
p<0.04^{\mathrm{b}, \mathrm{c}}\end{array}$ & $\begin{array}{l}\text { Serum data } \\
Y \\
Y\end{array}$ \\
\hline $\begin{array}{l}{ }^{a} \text { Primary clinical } \\
{ }^{b} \text { No test statistic providec } \\
\end{array}$ & & & & & & \\
\hline
\end{tabular}

\section{Bipolar disorder}

- There is insufficient evidence to determine efficacy. Of the three studies published with children with bipolar disorder, the only RCT [54] used alpha-linoleic acid (ALA); patients taking the ALA supplement were significantly more compliant but did not exhibit a significant conversion rate of ALA to EPA. Only the subgroup of subjects who acquired an EPA serum level of $0.8 \%$ or higher exhibited a clinical response to the treatment. Since conversion from ALA to EPA was limited and required a minimum of 1012 capsules/day, the authors concluded that an ALA treatment regimen is impractical. The two prospective open-label trials $[55,56]$ provide suggestive, non-significant evidence of reduction and remission of symptoms.

\section{Clinical evidence}

\begin{tabular}{|c|c|c|c|c|c|c|}
\hline Study & Type & $N$ & Daily dose/length & Outcome $^{a}$ & Statistic ${ }^{a}$ & $\begin{array}{c}\text { Serum } \\
\text { data }\end{array}$ \\
\hline $\begin{array}{l}\text { [54] Gracious } \\
\text { et al., } \\
2010\end{array}$ & RCT & 48 & $\begin{array}{l}4 \text { mo.: } 530-6,600 \mathrm{mg} \\
\text { ALA (titrated) }\end{array}$ & Compliance & $\begin{array}{c}\text { Breslow } X^{2}(1)= \\
4.17 \\
p=0.041^{\mathrm{e}}\end{array}$ & $Y$ \\
\hline
\end{tabular}




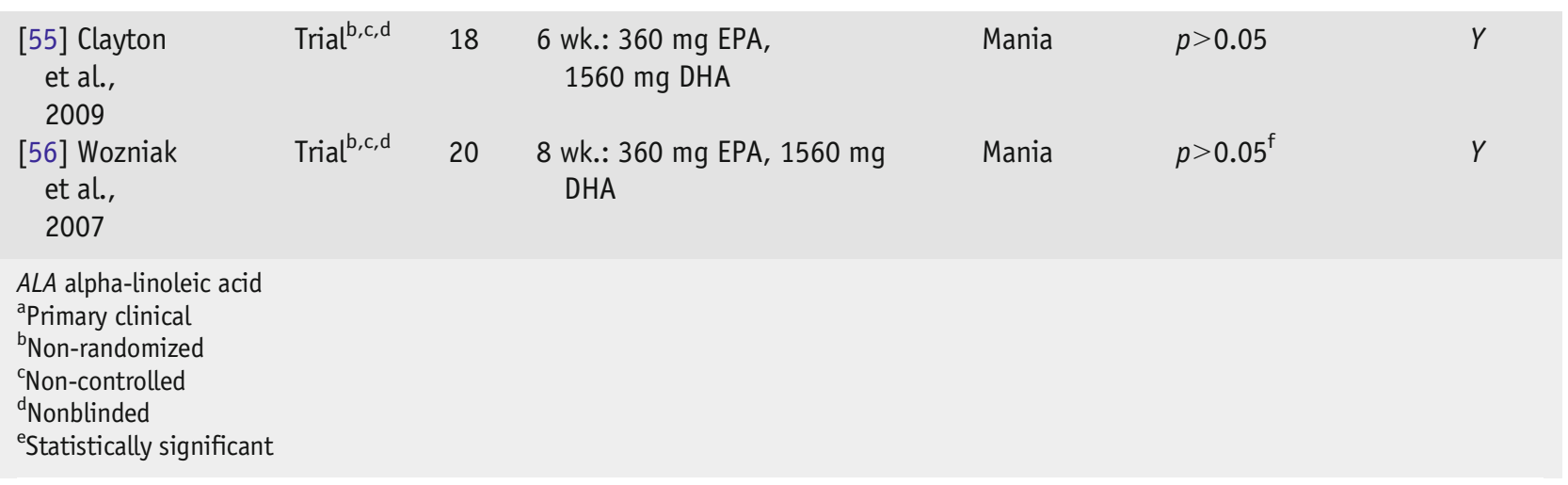

\section{Depression}

- There is insufficient evidence to determine efficacy. Of the three studies published with children with depression, one small RCT [57] observed a clinically meaningful treatment effect and the two open-label studies $[58,59 \bullet]$ provide statistically significant suggestive evidence of a clinical response. A postmortem study of prefrontal cortices of adolescent suicide victims did not find DHA tissue deficits compared to agematched controls [60].

\section{Clinical evidence}

\begin{tabular}{|c|c|c|c|c|c|c|}
\hline Study & Type & $N$ & Daily dose/length & Outcome $^{a}$ & Statistic ${ }^{a}$ & $\begin{array}{c}\text { Serum } \\
\text { data }\end{array}$ \\
\hline $\begin{array}{l}\text { [57] Nemets et al., } \\
2006\end{array}$ & RCT & 28 & $\begin{array}{l}4 \mathrm{mo} .: 400 \mathrm{mg} \text { EPA, } 200 \mathrm{mg} \\
\text { DHA }\end{array}$ & $\begin{array}{l}\text { Depressive } \\
\text { affect }\end{array}$ & $\begin{array}{l}F=12.2 \\
\quad p<0.003\end{array}$ & $N$ \\
\hline
\end{tabular}

\section{Discussion/conclusion}

Omega-3 fatty acids have been explored for a wide-ranging variety of child/ adolescent disorders.

In general, effects have been small, but in several disorders significant. The small benefit is balanced by an even smaller safety risk as long as only mercuryfree oils are used in moderate doses (not more than 2-3 g/day), and intake of antioxidant vitamins is adequate. Theoretically alpha-linolenic acid (ALA), the 18-carbon precursor, should be elongated and further desaturated to make the 20-carbon eicosapentaenoic (EPA and 22-carbon docosahexaenoic (DHA)) acids that are critical to brain health and function. However, one of the genetic predispositions to mental disorders may be a deficient fatty acid desaturase enzyme necessary for the anabolism of EPA and DHA from ALA. Indeed, 
what few data are available suggest that ALA does not work as well as $\mathrm{EPA} / \mathrm{DHA}$. At least one meta-analysis suggested that EPA is the most important of all to ingest. This is the precursor not only of DHA, but also of anti-inflammatory prostaglandins and other "eicosanoids."

All things considered, including general health, we recommend modest doses of EPA/DHA (up to a gram per day) if the child does not eat oily wild ocean fish three times a week. This should be coupled with adequate intake of vitamins and minerals. Higher doses may be tried for disorders that have some evidence of efficacy. Three months are needed for an adequate trial.

\section{Compliance with Ethics Guidelines}

\section{Conflict of Interest}

Barbara L. Gracious and Lipi Gupta declare that they have no conflict of interest. Dr. Arnold has received research funding from Curemark, Forest, Lilly, Neuropharm, Novartis, Noven, Shire, and YoungLiving (as well as NIH and Autism Speaks) and has consulted with or been on advisory boards for Gowlings, Neuropharm, Novartis, Noven, Organon, Otsuka, Pfizer, Roche, Seaside Therapeutics, Sigma Tau, Shire, and Tris Pharma and received travel support from Noven.

\section{Human and Animal Rights and Informed Consent}

Among cited articles where one of the authors of the current report were authors, local Institutional Review Board approval was obtained and maintained for studies where human (or animal) subjects research was performed.

\section{References and Recommended Reading}

Papers of particular interest, published recently, have been highlighted as:

- Of importance,

- Of major importance

1. Belfer ML. Child and adolescent mental disorders: the magnitude of the problem across the globe. J Child Psychol Psychiatry. 2008;49:226-36.

2. Harris WS. Expert opinion: omega-3 fatty acids and bleeding-cause for concern? Am J Cardiol. 2007;99(6):S44-6.

3. Riediger ND, Othman RA, Suh M, Moghasdiasian MH. A systemic review of the roles of n-3 fatty acids in health and disease. J Am Diet Assoc. 2009;109(4):688-79.

4. Freeman MP, Hibbeln JR, Wisner KL, Davis JM, Mischoulon D, Peet $M$, et al. Omega-3 fatty acids: evidence basis for treatment and future research in psychiatry. J Clin Psychiatry.2006;67(12):1954-67.

5. McNamara RK, Carlson SE. Role of omega-3 fatty acids in brain development and function: Potential implications for the pathogenesis and prevention of psychopathology. Prostaglandins Leukot Essent Fat Acids. 2006;75:329-49.
This review summarizes historical importance of omega-3 fatty acids in neurodevelopment, describing brain composition (including neuronal membrane properties), CNS accrual, and effects of deficit states in animals and humans on neuronal functioning, cellular signaling, and outcomes, including risk for psychopathology including ADHD and schizophrenia.

6.• Blasbalg TL, Hibbeln JR, Ramsden CE, et al. Changes in consumption of omega-3 and omega- 6 fatty acids in the United States during the 20th century. Am J Clin Nutr. 2011;93:950-62.

This paper provides strong epidemiologic evidence to support the hypothesis that dietary changes in omega fatty acid intake contribute to the pathogenesis of psychopathology.

7. Sublette ME, Galfalvy HC, Hibbeln JR, et al. Polyunsaturated fatty acid associations with dopaminergic indices in major depressive disorder. Int J Neuropsychopharmacol. 2014;17(03):383-91. 
8. Mitchell RHB, Goldstein BI. Inflammation in Children and Adolescents with Neuropsychiatric Disorders: a systemic review. JAACAP. 2014;53(3):274-96.

9.• Hibbeln JR, David JM, Steer C, Emmett P, Rogers I, Williams C, et al. Maternal seafood consumption in pregnancy and neurodevelopmental outcomes in childhood (ALSPAC study): an observational cohort study. Lancet. 2007;369(9561):578-85.

This large epidemiologic study confirms the importance of adequate omega-3 intake on normal human developmental outcomes, especially intellect.

10. Sinn N, Bryan J, Wilson C. Cognitive effects of polyunsaturated fatty acids in children with attention deficit hyperactivity disorder symptoms: a randomised controlled trial. Prostaglandins Leukot Essent Fatty Acids. 2008;78(4-5):311-26.

11. Sinn N, Bryan J. Effect of supplementation with polyunsaturated fatty acids and micronutrients on learning and behavior problems associated with child ADHD. J Dev Behav Pediatr. 2007;28(2):82-91.

12. Widenhorn-Muller K, Schwanda S, Scholz E, Spitzer M, Bode $\mathrm{H}$. Effect of supplementation with long-chain omega-3 polyunsaturated fatty acids on behavior and cognition in children with attention deficit/ hyperactivity disorder (ADHD): A randomized placebo-controlled intervention trial. Prostaglandins Leukot Essent Fat Acids. 2014.

13. Milte CM, Parletta N, Buckley JD, Coates AM, Young RM, Howe PRC. Eicosapentaenoic and docosahexaenoic acids, cognition, and behavior in children with attention-deficit/hyperactivity disorder: a randomized controlled trial. Nutrition.

2012;28(6):670-7.

14. Milte CM, Parletta N, Buckley JD, Coates AM, Young RM, Howe PRC. Increased erythrocyte eicosapentaenoic acid and docosahexaenoic acid are associated with improved attention and behavior in children with ADHD in a randomized controlled three-way crossover trial. J Atten Disord. 2013;1087054713510562.

15. Joshi K, Lad S, Kale M, Patwardhan B, Mahadik SP, Patni B, et al. Supplementation with flax oil and vitamin $\mathrm{C}$ improves the outcome of attention deficit hyperactivity disorder (ADHD). Prostaglandins Leukot Essent Fat Acids. 2006;74(1):17-21.

16. Belanger SA, Vanasse M, Spahis S, Sylvestre MP, Lippe S, L'Heureux F, et al. Omega-3 fatty acid treatment of children with attention-deficit hyperactivity disorder: a randomized, double-blind, placebo-controlled study. Paediatr Child Health. 2009;14(2):89-98.

17. Johnson M, Mansson JE, Ostlund S, Fransson G, Areskoug B, Hjalmarsson K, et al. Fatty acids in ADHD: plasma profiles in a placebo-controlled study of omega $3 / 6$ fatty acids in children and adolescents. Atten Defic Hyperact Disord. 2012;4(4):199-204.

18. Johnson M, Ostlund S, Fransson G, Kadesjo B, Gillberg C. Omega-3/omega-6 fatty acids for attention deficit hyperactivity disorder: a randomized placebocontrolled trial in children and adolescents. J Atten Disord. 2009;12(5):394-401.
19. Gustafsson PA, Birberg-Thornberg U, Duchen K, Landgren M, Malmberg K, Pelling H, et al. EPA supplementation improves teacher-rated behaviour and oppositional symptoms in children with ADHD. Acta Paediatr. 2010;99(10):1540-9.

20. Manor I, Magen A, Keidar D, Rosen S, Tasker H, Cohen $\mathrm{T}$, et al. Safety of phosphatidylserine containing omega3 fatty acids in ADHD children: a double-blind placebo-controlled trial followed by an open-label extension. Eur Psychiatry. 2013;28(6):386-91.

21. Manor I, Magen A, Keidar D, Rosen S, Tasker H, Cohen $\mathrm{T}$, et al. The effect of phosphatidylserine containing omega3 fatty-acids on attention-deficit hyperactivity disorder symptoms in children: a double-blind placebo-controlled trial, followed by an open-label extension. Eur Psychiatry. 2012;27(5):335-42.

22. Raz R, Carasso RL, Yehuda S. The influence of shortchain essential fatty acids on children with attentiondeficit/hyperactivity disorder: a double-blind placebocontrolled study. J Child Adolesc Psychopharmacol. 2009;19(2):167-77.

23. Arnold LE, Kleykamp D, Votolato NA, Taylor WA, Kontras SB, Tobin K. Gamma-linolenic acid for attention-deficit hyperactivity disorder: placebocontrolled comparison to D-amphetamine. Biol Psychiatry. 1989;25(2):222-8.

24. Aman MG, Mitchell EA, Turbott SH. The effects of essential fatty acid supplementation by Efamol in hyperactive children. J Abnorm Child Psychol. 1987;15(1):75-90.

25. Barragán E, Breuer D, Döpfner M. Efficacy and safety of omega-3/6 fatty acids, methylphenidate, and a combined treatment in children with ADHD. J Atten Disord. 2014;1087054713518239.

26. Millichap JG. $\Omega-3 / 6$ Fatty Acids, Methylphenidate, and Combined ADHD Therapy. AAP Grand Rounds. 2014;31(6).

27. Hariri M, Djazayery A, Djalali M, Saedisomeolia A, Rahimi A, Abdolahian E. Effect of n-3 supplementation on hyperactivity, oxidative stress and inflammatory mediators in children with attention-deficithyperactivity disorder. Malays J Nutr. 2012;18(3):32935.

28. Perera H, Jeewandara KC, Seneviratne S, Guruge C. Combined $\omega 3$ and $\omega 6$ supplementation in children with attention-deficit hyperactivity disorder (ADHD) refractory to methylphenidate treatment a doubleblind, placebo-controlled study. J Child Neurol. 2012;27(6):747-53.

29. Kirby A, Woodward A, Jackson S, Wang Y, Crawford MA. A double-blind, placebo-controlled study investigating the effects of omega- 3 supplementation in children aged 8-10 years from a mainstream school population. Res Dev Disabil. 2010;31(3):718-30.

30. Vaisman N, Kaysar N, Zaruk-Adasha Y, Pelled D, Brichon G, Zwingelstein G, et al. Correlation between changes in blood fatty acid composition and visual sustained attention performance in children with 
inattention: effect of dietary n-3 fatty acids containing phospholipids. Am J Clin Nutr. 2008;87(5):1170-80.

31. Voigt RG, Llorente AM, Jensen CL, Fraley JK, Berretta MC, Heird WC. A randomized, double-blind, placebocontrolled trial of docosahexaenoic acid supplementation in children with attention-deficit/hyperactivity disorder. J Pediatr. 2001;139(2):189-96.

32. Hirayama S, Hamazaki T, Terasawa K. Effect of docosahexaenoic acid-containing food administration on symptoms of attention-deficit/hyperactivity disorder-a placebo-controlled double-blind study. Eur J Clin Nutr. 2004;58(3):467-73.

33. Richardson AJ, Puri BK. A randomized double-blind, placebo-controlled study of the effects of supplementation with highly unsaturated fatty acids on ADHDrelated symptoms in children with specific learning difficulties. Prog Neuropsychopharmacol Biol Psychiatry. 2002;26(2):233-9.

34. Stevens L, Zhang W, Peck L, Kuczek T, Grevstad N, Mahon A, et al. EFA supplementation in children with inattention, hyperactivity, and other disruptive behaviors. Lipids. 2003;38(10):1007-21.

35. Gabbay V, Babb JS, Klein RG, Panzer AM, Katz Y, Alonso CM, et al. A double-blind, placebo-controlled trial of omega-3 fatty acids in Tourette's disorder. Pediatrics. 2012;129(6):e1493-500.

36. Richardson AJ, Montgomery P. The Oxford-Durham study: a randomized, controlled trial of dietary supplementation with fatty acids in children with developmental coordination disorder. Pediatrics.

2005;115(5):1360-6.

37. Stordy BJ. Dark adaptation, motor skills, docosahexaenoic acid, and dyslexia. Am J Clin Nutr. 2000;71(1 Suppl):323s-6s.

38. Richardson AJ, Burton JR, Sewell RP, Spreckelsen TF, Montgomery P. Docosahexaenoic acid for reading, cognition and behavior in children aged 7-9 years: a randomized, controlled trial (the DOLAB Study). PLoS ONE. 2012;7(9):e43909.

39. Osendarp SJ, Baghurst KI, Bryan J, Calvaresi E, Hughes $\mathrm{D}$, Hussaini M, et al. Effect of a 12-mo micronutrient intervention on learning and memory in wellnourished and marginally nourished school-aged children: 2 parallel, randomized, placebo-controlled studies in Australia and Indonesia. Am J Clin Nutr. 2007;86(4):1082-93.

40. Kairaluoma L, Narhi V, Ahonen T, Westerholm J, Aro $M$. Do fatty acids help in overcoming reading difficulties? A double-blind, placebo-controlled study of the effects of eicosapentaenoic acid and carnosine supplementation on children with dyslexia. Child Care Health Dev. 2009;35:112-9.

41. Ryan AS, Nelson EB. Assessing the effect of docosahexaenoic acid on cognitive functions in healthy, preschool children: a randomized, placebocontrolled, double-blind study. Clin Pediatr. 2008.

42. Voigt RG, Mellon MW, Katusic SK, Weaver AL, Matern D, Mellon B, et al. Dietary docosahexaenoic acid supplementation in children with autism. J Pediatr Gastroenterol Nutr. 2014;58(6):715-22.

43. Bent S, Hendren RL, Zandi T, Law K, Choi JE, Widjaja F, et al. Internet-based, randomized, controlled trial of omega-3 fatty acids for hyperactivity in autism. J Am Acad Child Adolesc Psychiatry. 2014;53(6):658-66.

44. Bent S, Bertoglio K, Ashwood P, Bostrom A, Hendren RL. A pilot randomized controlled trial of omega-3 fatty acids for autism spectrum disorder. J Autism Dev Disord. 2011;41(5):545-54.

45. Johnson CR, Handen BL, Zimmer M, Sacco K. Polyunsaturated fatty acid supplementation in young children with autism. J Dev Phys Disabil. 2010;22(1):1-10.

46. Meguid NA, Atta HM, Gouda AS, Khalil RO. Role of polyunsaturated fatty acids in the management of Egyptian children with autism. Clin Biochem. 2008;41(13):1044-8.

47. Amminger GP, Berger GE, Schafer MR, Klier C, Friedrich $\mathrm{MH}$, Feucht M. Omega-3 fatty acids supplementation in children with autism: a double-blind randomized, placebo-controlled pilot study. Biol Psychiatry. 2007;61(4):551-3.

48. Meiri G, Bichovsky Y, Belmaker RH. Omega 3 fatty acid treatment in autism. J Child Adolesc Psychopharmacol. 2009;19(4):449-51.

49.• Amminger GP, Schafer MR, Papageorgiou K, Klier CM, Cotton SM, Harrigan SM, et al. Long-chain omega-3 fatty acids for indicated prevention of psychotic disorders: a randomized, placebo-controlled trial. Arch Gen Psychiatry. 2010;67(2):146-54.

This 12-week trial in 81 young people aged 13-25 with subthreshold psychosis provided the first clear evidence that omega-3 fatty acids, given in the early stages of psychotic illness development, prevent progression, and potentially the ultimate burdens associated with psychotic disorders. Significant effects were noted as soon as 1 month for positive symptoms, with improvement in negative symptoms noted at 3 months in a post-hoc analysis (PMID 237780322014).

50. Amminger GP, Chanen AM, Ohmann S, Klier CM, Mossaheb N, Bechdolf A, et al. Omega-3 fatty acid supplementation in adolescents with borderline personality disorder and ultra-high risk criteria for psychosis: a post hoc subgroup analysis of a double-blind, randomized controlled trial. Can J Psychiatry. 2013;58(7):402-8.

51. Smesny S, Milleit B, Hipler UC, Milleit C, Schafer MR, Klier CM, et al. Omega-3 fatty acid supplementation changes intracellular phospholipase A2 activity and membrane fatty acid profiles in individuals at ultrahigh risk for psychosis. Mol Psychiatry. 2014;19(3):317-24.

52. Dean AJ, Bor W, Adam K, Bowling FG, Bellgrove MA. A randomized, controlled, crossover trial of fish oil treatment for impulsive aggression in children and adolescents with disruptive behavior disorders. J Child Adolesc Psychopharmacol. 2014;24(3):140-8.

53. Itomura M, Hamazaki K, Sawazaki S, Kobayashi M, Terasawa K, Watanabe S, et al. The effect of fish oil on physical aggression in schoolchildren-a randomized, 
double-blind, placebo-controlled trial. J Nutr Biochem. 2005;16(3):163-71.

54. Gracious BL, Chirieac MC, Costescu S, Finucane TL, Youngstrom EA, Hibbeln JR. Randomized, placebocontrolled trial of flax oil in pediatric bipolar disorder. Bipolar Disord. 2010;12(2):142-54.

55. Clayton EH, Hanstock TL, Hirneth SJ, Kable CJ, Garg ML, Hazell PL. Reduced mania and depression in juvenile bipolar disorder associated with long-chain omega-3 polyunsaturated fatty acid supplementation. Eur J Clin Nutr. 2009;63(8):1037-40.

56. Wozniak J, Biederman J, Mick E, Waxmonsky J, Hantsoo L, Best C, et al. Omega-3 fatty acid monotherapy for pediatric bipolar disorder: a prospective open-label trial. Eur Neuropsychopharmacol. 2007;17(6-7):440-7.

57. Nemets H, Nemets B, Apter A, Bracha Z, Belmaker RH. Omega-3 treatment of childhood depression: a controlled, double-blind pilot study. Am J Psychiatry. 2006;163(6):1098-100.

58. McNamara RK, Jandacek R, Rider T, Tso P, Chu WJ, Weber WA, et al. Effects of fish oil supplementation on prefrontal metabolite concentrations in adolescents with major depressive disorder: A preliminary H MRS study. Nutr Neurosci. 2014.
59.• McNamara RK, Strimpfel J, Jandacek R, Rider T, Tso P, Welge JA, et al. Detection and treatment of long-chain omega-3 fatty acid deficiency in adolescents with SSRIresistant major depressive disorder. PharmaNutrition. 2014;2(2):38-46.

This small, open-label 10-week trial of a low and high dose fish oil preparation found baseline RBC DHA deficits in all 20 teens; symptom remission occurred in $100 \%$ of those treated with the high-dose (16.2 g/day), providing strong support for larger definitive trials.

60. McNamara RK, Jandacek R, Rider T, Tso P, Dwivedi Y, Roberts RC, et al. Fatty acid composition of the postmortem prefrontal cortex of adolescent male and female suicide victims. Prostaglandins Leukot Essent Fat Acids. 2009;80(1):19-26.

61. Puri BK, Martins JG. Which polyunsaturated fatty acids are active in children with attention-deficit hyperactivity disorder receiving PUFA supplementation? A fatty acid validated meta-regression analysis of randomized controlled trials. Prostaglandins Leukot Essent Fat Acids (PLEFA). 2014;90(5):179-89.

This most recent meta-analysis notes modest evidence especially for GLA and EPA for inattentive symptoms in ADHD. They also reiterate concerns that some placebos may actually be psychoactive and therefore limit generalizability of findings. 